\title{
Taste quality and extinction of a conditioned taste aversion in rats
}

\author{
RICK A. BEVINS, HEATHER C. JENSEN, TODD S. HINZE, and JOYCE BESHEER \\ University of Nebraska, Lincoln, Nebraska
}

\begin{abstract}
Rats (Rattus norvegicus) that received a taste cue (saccharin, saline, quinine, or sucrose) paired with a lithium chloride ( $\mathrm{LiCl}$ ) injection displayed a robust decrease in consumption of that taste, relative to controls that had the taste unpaired with LiCl. Consumption of the paired taste increased with each nonreinforced presentation (i.e., extinction). After asymptotic extinction, rats that had had a $0.1 \%$ saccharin cue paired with $\mathrm{LiCl}$ consumed less of the saccharin solution than did controls. A similar data pattern was observed with a $10 \%$ sucrose solution. These results are consistent with the view that some aspect of the excitatory CS-US association remains after extinction. On the other hand, rats that had a bitter $(0.005 \%$ or $0.001 \%$ quinine) or salty ( $1 \%$ or $0.5 \%$ saline) solution paired with LiCl drank similar amounts of the fluid as controls after asymptotic extinction treatment. Together, these experiments suggest that a taste that is either sweet or preferred is required in order to demonstrate the chronic decrease in fluid consumption after extinction treatment. The data suggest that the conditioning experience prevents the later development of a preference for the sweet taste, rather than there being a retained aversion that suppresses fluid consumption.
\end{abstract}

Since Pavlov (1927), researchers have spent an enormous amount of effort elucidating the processes that modulate the acquisition, maintenance, and subsequent extinction of Pavlovian conditioning. There has been a recent surge of interest in the processes governing extinction (e.g., Bouton, 1991; Delamater, 1996; Rescorla, 1997; Robbins, 1990). To demonstrate extinction, one first establishes Pavlovian excitatory conditioning by pairing a relatively neutral stimulus (conditioned stimulus, or CS) with a somewhat more biologically relevant stimulus (unconditioned stimulus, or US). The CS comes to elicit a conditioned response (CR). Elicitation of a CR is taken as evidence for an excitatory association between the CS and the US. Extinction refers to the subsequent presentation of this CS without the US and the observation that the frequency or magnitude of the CR decreases toward a preconditioning baseline (see Miller \& Oberling, 1998, for an interesting discussion of the definition of extinction).

There are numerous hypotheses concerning the process(es) governing the decrease in conditioned responding with extinction. For example, Pavlov (1927) suggested that the excitatory association formed during conditioning remained intact and that extinction involved formation of an inhibitory association that competed with

This research and the preparation of this report was supported, in part, by grants from the Nebraska Department of Health and Human Services and the University of Nebraska-Lincoln Research Council. We are grateful to Chana Akins and John Ayres for their comments on an earlier version of this manuscript. Correspondence concerning this article should be addressed to R. A. Bevins, Nebraska Behavioral Biology Group, Department of Psychology, University of Nebraska, Lincoln, NE 68588-0308 (e-mail: rbevins l @unl.edu). the excitatory association. In contrast, Skinner (1938) argued against Pavlov's notions of an inhibitory association and described extinction as a "reciprocal process" (p. 61). That is, extinction reflected the loss of the excitatory association (see, also, Rescorla \& Wagner, 1972). More recent research and theorizing tend to agree with Pavlov's conclusion that the excitatory CS-US association remains, at least in part, intact after extinction. Such processes that account for loss of the CR while the excitatory CS-US association is left intact include decreased attention to the CS (Robbins, 1990), failure to retrieve memory of the excitatory association as a result of acquiring an independent memory for extinction (Bouton, 1991), and acquisition of an inhibitory association between the CS and the CR (Pavlov, 1927; Rescorla, 1993, 1997).

Rosas and Bouton (1996, Experiment 4), using a conditioned taste aversion preparation, demonstrated a very interesting effect that could be used to elucidate the processes underlying extinction of a taste aversion. In that experiment, one set of rats (paired) received a novel saccharin taste CS paired with an illness-inducing lithium chloride ( $\mathrm{LiCl}$ ) injection. The $\mathrm{LiCl}$ US did not follow subsequent presentations of the saccharin CS (i.e., extinction). As was expected, the paired rats showed a substantial decrease in saccharin intake on the first extinction trial (i.e., conditioned saccharin aversion). Saccharin intake increased across repeated extinction trials. Interestingly, even after eight extinction trials, the paired rats did not drink as much as the control rats that had equal exposure to the saccharin CS and the LiCl US in an unpaired fashion. Rosas and Bouton concluded that this betweengroup difference in saccharin intake was evidence that the excitatory association conditioned by the saccharinlithium pairing remained intact after extinction. For ease 
Table 1

Milliliter Intake ( $₫ 1$ SEM) of Fluids Across the Four Experiments

\begin{tabular}{lllccc}
\hline & & & \multicolumn{2}{c}{ Extinction Trial } \\
\cline { 5 - 6 } Experiment & Group & Water (Day 5) & Conditioning & First & Last \\
\hline $1(0.1 \%$ saccharin) & Unpaired & $25.4 \pm .94$ & $14.9 \pm .80$ & $14.4 \pm 1.28$ & $19.4 \pm 1.00$ \\
& Paired & $25.1 \pm .96$ & $15.8 \pm .51$ & $2.2 \pm .68^{*}$ & $14.8 \pm 1.50^{*}$ \\
$2 \mathrm{~A}(1 \%$ saline $)$ & Unpaired & $24.0 \pm 1.23$ & $17.6 \pm .75$ & $14.3 \pm 1.16$ & $16.6 \pm .99$ \\
& Paired & $23.9 \pm 1.21$ & $15.9 \pm .82$ & $1.8 \pm .79^{*}$ & $14.8 \pm 1.32$ \\
$2 \mathrm{~B}(0.5 \%$ saline $)$ & Unpaired & $17.4 \pm 1.28$ & $14.6 \pm .38$ & $15.5 \pm 1.0$ & $13.4 \pm .92$ \\
& Paired & $18.6 \pm .93$ & $16.6 \pm .94$ & $6.9 \pm 1.65^{*}$ & $15.4 \pm .91$ \\
$3 \mathrm{~A}(0.005 \%$ quinine) & Unpaired & $24.7 \pm .87$ & $5.0 \pm .61$ & $6.8 \pm 1.01$ & $11.9 \pm .81$ \\
& Paired & $25.8 \pm 1.94$ & $5.0 \pm .58$ & $1.2 \pm .20^{*}$ & $10.9 \pm .90$ \\
$3 \mathrm{~B}(0.001 \%$ quinine) & Unpaired & $27.1 \pm 1.14$ & $11.0 \pm .99$ & $7.9 \pm .60$ & $14.2 \pm .80$ \\
& Paired & $26.7 \pm 1.20$ & $12.3 \pm 89$ & $4.8 \pm .96^{*}$ & $14.6 \pm .87$ \\
$4(10 \%$ sucrose $)$ & Unpaired & $28.4 \pm .84$ & $17.3 \pm .79$ & $16.1 \pm 1.69$ & $21.4 \pm .92$ \\
& Paired & $28.8 \pm .68$ & $18.0 \pm .48$ & $1.0 \pm .41^{*}$ & $18.6 \pm .98^{*}$ \\
\hline
\end{tabular}

*Significant difference $(p<.05)$ from the unpaired group.

of presentation, throughout the present report, we will refer to this persistent decrease in taste consumption following taste-illness pairing as conditioned hypodipsia.

\section{EXPERIMENT 1}

Rosas and Bouton's (1996) demonstration of conditioned hypodipsia was the first such example in the literature (see Nolan et al., 1997). Thus, the main purpose of Experiment 1 was to replicate this phenomenon in our laboratory. The procedural details of that experiment were similar to those in Rosas and Bouton (1996, Experiment 4), except that we used male Sprague -Dawley rats rather than female Wistar rats. A second purpose of Experiment 1 was to assess whether the conditioned hypodipsia effect would transfer to a new taste after the extinction phase. To do so, we replaced the saccharin solution with a saline solution on the day after the last extinction trial.

\section{Method}

\section{Subjects and Apparatus}

The subjects were 24 male Sprague-Dawley rats (345-423 g) that had previously served in an unrelated experiment that did not involve water deprivation or novel tastes. The rats were housed individually in plastic tubs lined with wood chips in a colony on a 12:12-h light:dark cycle. All the phases of the present experiment were conducted during the light portion of this cycle. Food was available continuously in the home cage; fluid access was restricted to $1 \mathrm{~h}$ /day (see below). Fluids were presented in $50-\mathrm{ml}$ graduated drinking tubes that allowed consumption to be measured to the nearest milliliter. All the taste cues were mixed in distilled water. $\mathrm{LiCl}$, the emetic US, was mixed in distilled water. All the injections were intraperitoneal (i.p.).

\section{Procedure}

Water baseline. Water was removed $24 \mathrm{~h}$ before the start of the experiment. On Days 1-5, each rat had 30-min access to distilled water in a morning session and a similar $30-\mathrm{min}$ access period in the afternoon. There were always two daily fluid presentations, separated by $8 \mathrm{~h}$. Throughout the experiment, each presentation was $30 \mathrm{~min}$, and the fluid in the afternoon session was always distilled water.
Conditioning. Before conditioning, the rats were assigned to one of two groups (paired or unpaired; 12 rats per group), with the restriction that overall water consumption on Day 5 was statistically similar and experimental history was comparably represented in each group. In the morning session of Day 6 , the rats were given 30 $\min$ access to a $0.1 \%$ sodium saccharin solution (w/v). Following access to the saccharin solution, the rats assigned to the paired group were injected i.p. with $\mathrm{LiCl}(127.2 \mathrm{mg} / \mathrm{kg})$; the rats in the unpaired control group received an injection of distilled water. On Day 7 , the rats were allowed 30 -min access to water in the morning session. The unpaired control rats were injected with $\mathrm{LiCl}$ immediately after water access; the rats in the paired group were injected with distilled water. Day 8 was a recovery day, in which water was available in both sessions.

Extinction and transfer test. On Day 9, the saccharin solution was presented in the morning session without a subsequent injection of $\mathrm{LiCl}$ (i.e., extinction). Throughout the present report, termination of the extinction phase was determined by the following criteria: (1) There had to be a minimum of eight extinction trials (cf. Rosas \& Bouton, 1996, Experiment 4), and (2) the data pattern for the paired group had to be statistically stable, relative to the control condition, for at least 3 days. In the present experiment, these criteria were reached on the ninth extinction trial. The day after the last extinction trial included a transfer test. A $1 \%$ sodium chloride (saline) solution replaced the saccharin solution in the morning session.

Data analysis. Rosas and Bouton (1996) used a relative measure of intake that divided the morning saccharin intake by the afternoon water intake. A value greater than 1 was taken to indicate a preference for the saccharin solution. We could not use this proportion measure, because morning intake $(13.9 \pm 0.45 \mathrm{ml})$ of water in our laboratory is significantly greater than afternoon intake [11.4 \pm $0.42 \mathrm{ml} ; t(23)=4.40$ ]. Thus, any proportion above I may simply reflect the fact that more fluid is consumed in the morning session. We used an alternative within-subjects measure in which saccharin intake on a given extinction trial was converted to a percent change from initial saccharin consumption, using the following formula: (extinction intake - initial intake)/initial intake $\times 100$. This measure was employed so that the magnitudes of change in fluid consumption could be readily compared. Similar results were found with absolute intake of fluid as the dependent measure (see Table 1). A repeated measure analysis of variance (ANOVA) was used to analyze the extinction data. Group (paired vs. unpaired) was the between-groups factor, and percent change from initial saccharin intake on each extinction trial was the repeated measure. For a priori pairwise comparisons (e.g., milliliters of water intake on Day 5), we used $t$ tests. Post hoc comparisons prompted by a significant 


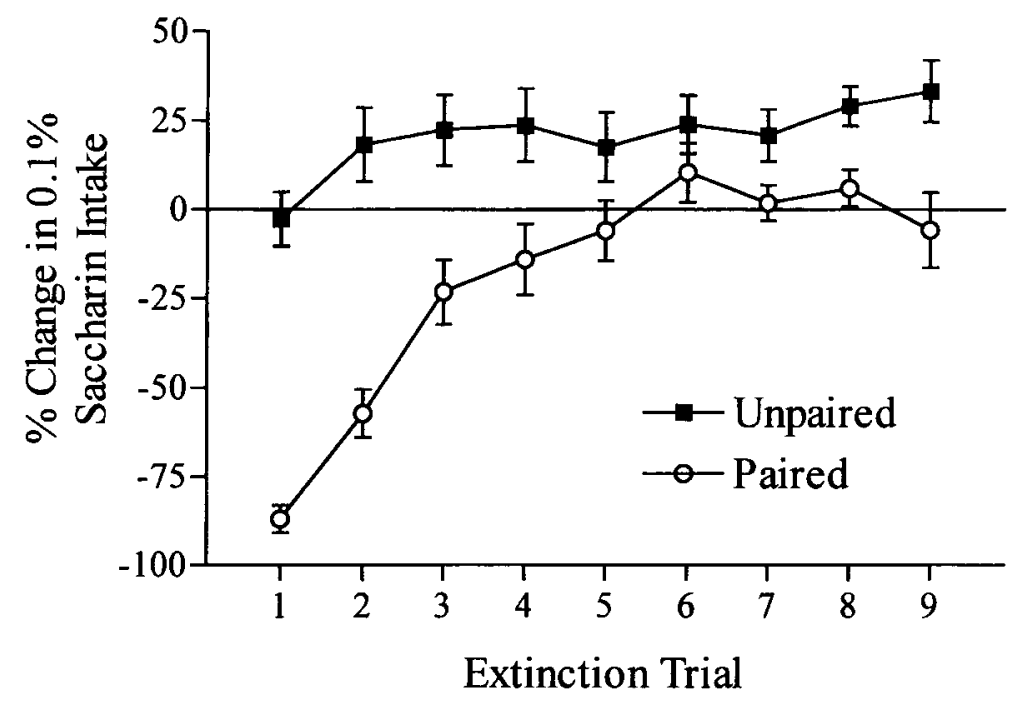

Figure 1. The mean percent change in consumption of a $0.1 \%$ sodium saccharin solution ( $\pm 1 S E M$ ) across the nine extinction trials for the paired and unpaired groups.

interaction employed pairwise $t$ tests. Statistical significance was declared with a two-tailed rejection region and an alpha of .05 for all the tests.

\section{Results and Discussion}

Table 1 displays fluid consumption for the last day of the water baseline phase, the conditioning trial, and the first and last extinction trials for each experiment in the present report. Water consumption for the paired and unpaired groups was similar on the day before conditioning $(t<1)$. Intake of the saccharin solution on the conditioning day was also comparable between groups $(t<1)$.

Figure 1 shows the percent change in saccharin consumption for each group across the nine extinction trials. Recall that the change in intake is relative to saccharin consumption on the conditioning day (i.e., first experience). Thus, a negative value denotes a decrease in saccharin intake, a zero indicates no change in consumption, and a positive value reflects an increase in consumption. There was an increase in saccharin consumption for the unpaired group in the early extinction trials. This increase appeared to be stable by Trial 2 . As was expected, the paired group showed a substantial decrease in saccharin consumption on the first extinction trial. This saccharin avoidance weakened across extinction trials. Although the rats in the paired group appeared to be approaching the intake level of the controls by Trial 6 , this trend reversed for the remaining extinction trials. The repeated measure ANOVA and subsequent post hoc contrasts supported these impressions. There were main effects of group $[F(1,22)=22.41]$ and of measure $[F(8,176)=$ $22.59]$. The group $\times$ measure interaction was also significant $[F(8,176)=8.31]$. Subsequent pairwise contrasts revealed lower saccharin intake for the paired group than for the unpaired group on Trials $1-4$ and Tri- als $7-9[t \mathrm{~s}(22) \geq 2.14]$. Table 1 shows saccharin consumption in milliliters for the first and the last extinction trial. The paired group drank less saccharin than the unpaired group on the first and last extinction trials $[t s(22) \geq 2.59]$. This result provides a replication of the conditioned hypodipsia effect demonstrated by Rosas and Bouton (1996) and extends the generality of the effect to include male Sprague-Dawley rats.

In the saline transfer test, the paired group drank less saline solution $(18.2 \pm 0.49 \mathrm{ml})$ than the unpaired controls [ $22 \pm 1.34 \mathrm{ml} ; t(22)=2.69]$. Presumably, the residual avoidance tendency of the saccharin solution seen in the paired group transferred to the saline solution. Others have reported generalization of conditioned taste aversion between saccharin and saline (e.g., Parker \& Revusky, 1982). Indeed, each taste has a sodium component (sodium saccharin vs. sodium chloride). In addition, saccharin also has a sweet and a bitter component (see Dess, 1993, for a recent review). Parker and Revusky also reported that a sucrose (sweet) aversion and a quinine (bitter) aversion both generalized to a saccharin solution. These observations pose several interesting questions as to the role of each taste quality in demonstrating the conditioned hypodipsia effect. Is one component more important than the other for expression? In the remaining experiments in the present report, we examine whether each primary taste component in saccharin (salty, bitter, and sweet), after being paired with $\mathrm{LiCl}$, will show the conditioned hypodipsia effect.

\section{EXPERIMENTS 2A AND 2B}

In Experiments $2 \mathrm{~A}$ and $2 \mathrm{~B}$, we examined whether a $1 \%$ saline solution (concentration in the transfer test of Experiment 1) or a $0.5 \%$ saline solution (a nonhypertonic 
concentration), respectively, paired with $\mathrm{LiCl}$, would result in conditioned hypodipsia.

\section{Method}

\section{Subjects and Apparatus}

The subjects were 37 male Sprague-Dawley rats similar to those described previously. The apparatus was unchanged, and the rats were housed in hanging stainless steel wire-mesh cages, rather than in the plastic tubs used in Experiment 1.

\section{Procedure}

Except where noted, the procedural details were identical to those of Experiment 1 . The taste CS was a 1\% sodium chloride solution ( $w / v)$ in Experiment $2 \mathrm{~A}$ and a $0.5 \%$ sodium chloride solution in Experiment 2B. The extinction criteria were met in 10 trials in both experiments; the transfer test was not conducted after the last extinction trial.

\section{Results and Discussion}

\section{Experiment 2A (1\% Saline)}

Water intake on Day 5 did not differ between the paired group $(n=10)$ and the unpaired group $(n=10$; $t<1$ ). Moreover, consumption of the $1 \%$ saline solution was similar for each group on the conditioning day $[t(18)=$ 1.53; see Table 1]. The percent change in saline consumption for each group across the 10 extinction trials is shown in Figure $2 \mathrm{~A}$. There was a main effect of measure $[F(9,162)=11.14]$ and a group $\times$ measure interaction $[F(9,162)=10.11]$. The main effect of group was not statistically significant $[F(1,18)=3.17]$. The paired group drank less saline than the unpaired group on Trials 1 and $2[t \mathrm{~s}(18) \geq 3.68]$. Consumption for each group was similar on the remaining extinction trials. Table 1 shows milliliters of saline consumed on the first and the last extinction trials. The paired group drank less saline than the unpaired group on the first extinction trial $[t(18)=8.95]$; there was no difference in saline consumption on the last extinction trial $[t(18)=1.09]$. Thus, a $1 \%$ saline solution paired with $\mathrm{LiCl}$ did not induce conditioned hypodipsia; group differences in intake were gone by the third extinction trial.

\section{Experiment 2B (0.5\% Saline)}

Water intake for the paired group $(n=9)$ and the unpaired group $(n=8)$ did not differ on Day $5(t<1)$. Intake of the $0.5 \%$ saline solution was statistically similar for each group on the conditioning day $[t(15)=1.81$; see Table 1]. Figure 2B shows the percent change in saline consumption for each group across the 10 extinction trials. The data pattern was very similar to that found in Experiment $2 \mathrm{~A}$ with the higher concentration of saline. There was a main effect of group $[F(1,15)=10.71]$, a main effect of measure $[F(9,135)=4.62]$, and a group $\times$ measure interaction $[F(9,135)=8.61]$. The paired group drank significantly less saline than the unpaired group on Trials 1,2 , and 4 [ $t \mathrm{~s}(15) \geq 2.43]$. Consumption for each group was similar on Trial 3 and on the last six extinction trials. Table 1 shows milliliters of saline consumed on the first and the last extinction trials. The paired group drank less saline than the unpaired group on the first extinction trial $[t(15)=4.32]$; there was no difference in saline consumption on the last extinction trial $[t(15)=1.59]$.

\section{EXPERIMENTS 3A AND 3B}

We did not find the conditioned hypodipsia effect with a hypertonic ( $1 \%)$ and nonhypertonic $(0.5 \%)$ concentration of saline. These results suggest that the primary taste of salt, unlike the more complex taste of saccharin, is not capable of maintaining conditioned hypodipsia under the present experimental conditions. However, one might consider that a bitter taste is essential for the conditioned hypodipsia effect. Bitter tastes, which are associated with poisons in the evolutionary history of the rat, readily elicit avoidance tendencies without explicit training. This unconditioned avoidance tendency may predispose rats to persistently avoid illness-paired bitter tastes, even if that taste has later been experienced numerous times without illness. Experiments $3 \mathrm{~A}$ and $3 \mathrm{~B}$ addressed this issue by using a predominantly bitter taste (i.e., a quinine solution). If our intuition is correct, rats that have the quinine solution paired with $\mathrm{LiCl}$ should show robust conditioned hypodipsia. As will be described later, the rats initially consumed a small amount of the $0.005 \%$ quinine solution in Experiment 3A. We decreased the quinine concentration in Experiment $3 B$, in order to increase intake.

\section{Method}

\section{Subjects and Apparatus}

The subjects were 44 male Sprague-Dawley rats similar to those described previously (Experiment $3 \mathrm{~A}, n=20$; Experiment $3 \mathrm{~B}, n=$ 24). The apparatus was unchanged. The rats in Experiment $3 \mathrm{~A}$ were housed in hanging stainless steel wire-mesh cages; the rats in Experiment $3 \mathrm{~B}$ were housed in plastic tubs.

\section{Procedure}

Except where noted, the procedural details of Experiments $3 \mathrm{~A}$ and $3 \mathrm{~B}$ were identical to those of Experiment $2 \mathrm{~A}$. The taste $\mathrm{CS}$ in Experiment $3 \mathrm{~A}$ was a $0.005 \%$ quinine sulfate solution $(\mathrm{w} / \mathrm{v})$, and in Experiment $3 \mathrm{~B}$, the taste was a $0.001 \%$ quinine solution. The extinction criteria were met in 9 trials for Experiment $3 \mathrm{~A}$ and in 11 trials for Experiment 3B. In Experiment 3B, there was no water recovery day before the first extinction trial.'

\section{Results and Discussion}

\section{Experiment 3A (0.005\% Quinine)}

Water intake for the paired and unpaired groups did not differ on Day $5(t<1)$. Intake of the $0.005 \%$ quinine solution was similar for each group on the conditioning day $(t<1$; see Table 1$)$. Figure 3A shows the percent change in quinine consumption for each group across the nine extinction trials. There was a main effect of measure $[F(8,144)=14.70]$ and a significant group $\times$ measure interaction $[F(8,144)=5.82]$. The main effect of group was not significant $[F(1,18)=2.46]$. Quinine intake in the paired group was lower than in the unpaired group for the first three extinction trials $[t \mathrm{~s}(18) \geq 2.48]$. Con- 

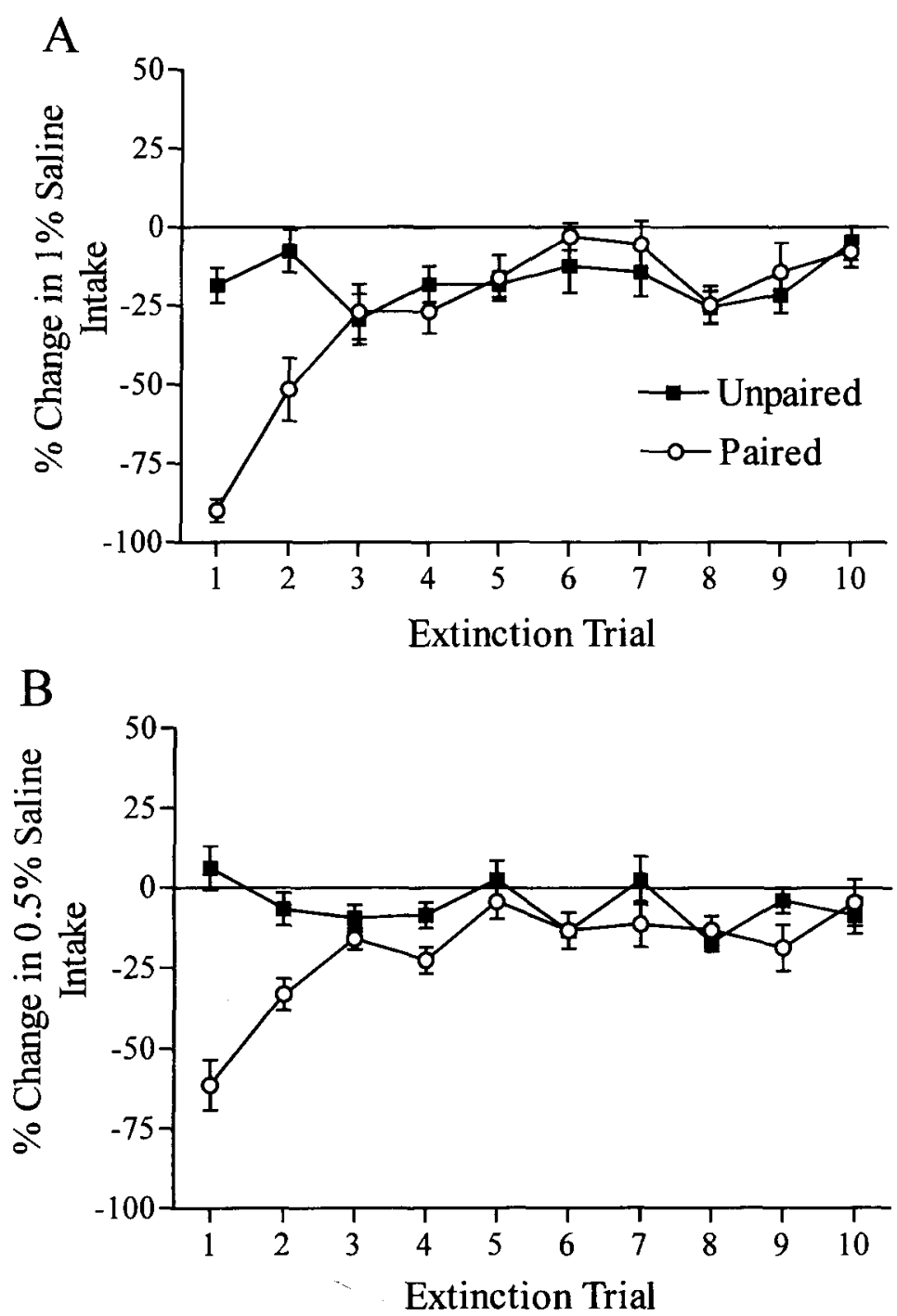

Figure 2. The mean percent change in consumption of a sodium chloride (saline) solution across the extinction trials for the paired and unpaired groups of Experiments $2 A$ and $2 B$ (panels $A$ and $B$, respectively).

sumption was similar for the remaining six trials. Table 1 shows milliliters of quinine consumed on the first and the last extinction trials. The paired group drank less quinine than the unpaired group on the first extinction trial $[t(18)=$ $5.44]$. There was no difference in quinine consumption by the last extinction trial $(t<1)$.

The conditioned hypodipsia effect seen with saccharin in Experiment 1 was not found in the present experiment with a $0.005 \%$ quinine solution. This result suggests that the bitter component of saccharin may not be required for conditioned hypodipsia to be demonstrated. The results of Experiments $2 \mathrm{~A}$ and $2 \mathrm{~B}$, moreover, suggest that the salt component was not required. Perhaps the effect is dependent on the presence of the sweet component of saccharin (i.e., the remaining primary taste quality in saccharin). Before discussing this notion in more detail, we must as- sess an alternative explanation for the quinine results. Initial intake of $0.005 \%$ quinine $(5 \mathrm{ml})$ was lower than that for the $0.1 \%$ saccharin $(16-\mathrm{ml})$, the $1 \%$ saline $(16-\mathrm{ml})$, or the $0.5 \%$ saline $(17-\mathrm{ml})$ solutions in the previous experiments. One could argue that the lower intake (decreased exposure to the CS) may have resulted in a weaker excitatory association between the taste CS and the emetic US (but see Bennett, Tremain, \& MacKintosh, 1996). In order to increase initial intake of quinine, we lowered the quinine concentration to $0.001 \%$ in Experiment 3B.

\section{Experiment 3B (0.001\% Quinine)}

Water intake for the paired and unpaired groups was similar on Day $5(t<1$; see Table 1$)$. Intake of the $0.001 \%$ quinine solution on the conditioning day was also similar for each group $(t<1)$. Importantly, initial intake of the 


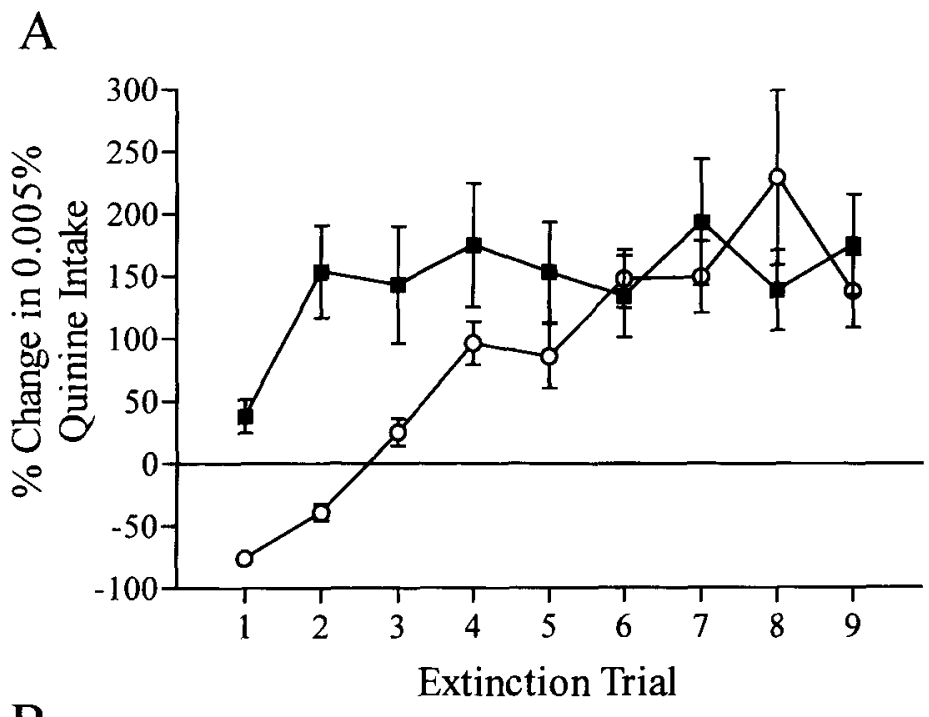

B

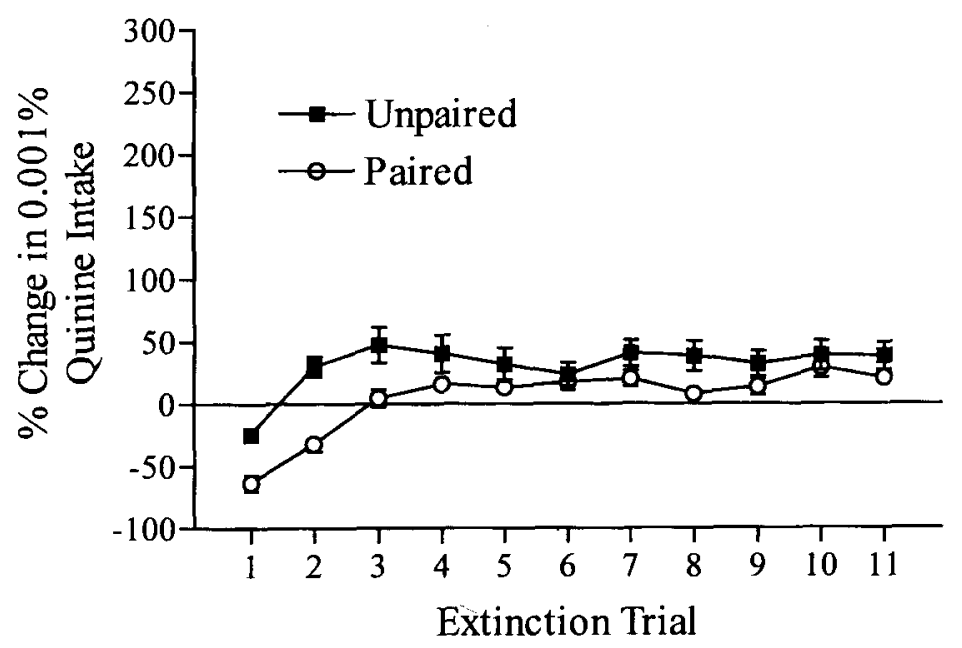

Figure 3. The mean percent change in consumption of a quinine sulfate solution across the extinction trials for the paired and unpaired groups of Experiments $3 A$ and $3 B$ (panels $A$ and $B$, respectively).

$0.001 \%$ quinine solution $(11-12 \mathrm{ml})$ was more than double that for the $0.005 \%$ solution $(5 \mathrm{ml})$. Figure $3 \mathrm{~B}$ shows the percent change in quinine consumption for each group across the 11 extinction trials. The data pattern was similar to that in the previous quinine experiment. There were main effects of group $[F(1,22)=5.96]$ and of measure $[F(10,220)=35.52]$. The group $\times$ measure interaction was also significant $[F(10,220)=4.79]$. Quinine intake in the paired group was lower than that in the unpaired group for the first 3 extinction trials and on Trial 8 $[t \mathrm{~s}(22) \geq 2.30]$. Consumption was similar on the remaining trials. Table 1 shows milliliters of quinine consumed on the first and the last extinction trials. The paired group drank less quinine than the unpaired group on the first extinction trial $[t(22)=2.78]$. There was no difference in quinine consumption on the last extinction trial $(t<1)$.
The combined results of Experiments $3 \mathrm{~A}$ and $3 \mathrm{~B}$ strongly suggest that the conditioned hypodipsia effect does not occur with a bitter taste. We failed to find conditioned hypodipsia with two different concentrations of quinine. An explanation based on a weak association resulting from low quinine intake seems unlikely for several reasons. First, quinine intake was more than doubled in Experiment 3B, and the overall data pattern did not differ dramatically from that with the less-consumed concentration of Experiment 3A. Second, the importance of amount consumed seems to depend on the nature of the taste CS. For example, the degree of taste aversion tends to increase with amount of intake if a complex taste (e.g., saccharin) is used (Barker, 1976; Bennett et al., 1996; Bond \& Di Giusto, 1975). In contrast, Bennett et al. found that the strength of a conditioned taste aversion does not vary with 


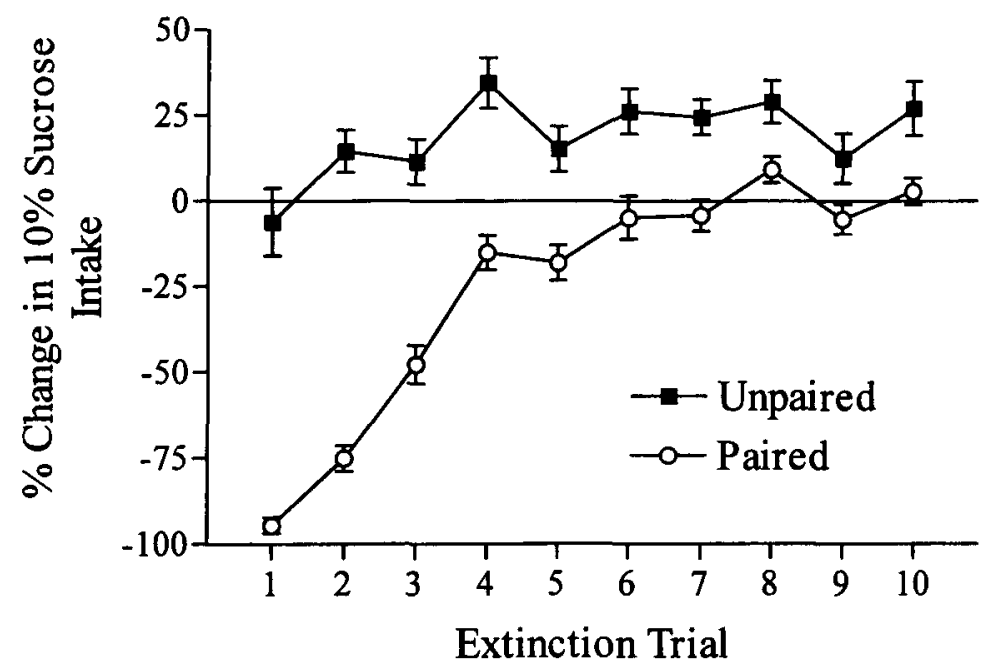

Figure 4. The mean percent change in consumption of a $10 \%$ sucrose solution $( \pm 1 S E M)$ across the 10 extinction trials for the paired and unpaired groups.

the amount of fluid consumed if the taste CS is simple (e.g., sucrose or hydrochloric acid). Although Bennett et al. did not examine a quinine CS, presumably quinine would be considered a simple primary taste.

\section{EXPERIMENT 4}

A saccharin taste supported conditioned hypodipsia. Saccharin is a complex taste cue that contains the primary taste qualities of salty, bitter, and sweet. Predominately salty tastes (Experiments $2 \mathrm{~A}$ and $2 \mathrm{~B}$ ) and predominately bitter tastes do not appear to be necessary for the conditioned hypodipsia effect. In Experiment 4, we assessed whether the sweet quality was sufficient to demonstrate conditioned hypodipsia. To do so, we used a $10 \%$ sucrose solution as the taste CS.

\section{Method}

The subjects were 24 male Sprague-Dawley rats similar to those described previously. The apparatus was unchanged, and the rats were housed in wire-mesh cages. The procedural details resembled those of Experiment $2 \mathrm{~A}$, except that a $10 \%$ sucrose solution (w/v) was employed as the taste $\mathrm{CS}$. The extinction criteria were reached after 10 extinction trials.

\section{Results and Discussion}

Water intake for the paired and unpaired groups was statistically similar on the day before conditioning $(t<1$; Table 1). Also, there was no difference in sucrose intake on the subsequent conditioning day $(t<1)$. Figure 4 shows the percent change in sucrose consumption for each group across the 10 extinction trials. For the unpaired group, sucrose intake increased in the early portion of the extinction phase, and that increase was maintained throughout. The paired group showed a robust sucrose aversion on the first extinction test. Sucrose intake increased across extinction trials. However, intake of the sucrose solution for the paired group never reached the level of that for the control group. There were main effects of group $[F(1,22)=48.25]$ and of measure $[F(9,198)=$ 59.13] and a group $\times$ measure interaction $[F(9,198)=$ 20.88]. Subsequent comparisons revealed that sucrose intake for the paired group was lower than that for the unpaired group at each extinction trial $[t s(22) \geq 2.10]$. Table 1 shows milliliters of sucrose consumed on the first and the last extinction trials. The paired group drank less sucrose than the unpaired group on the first and last extinction trials $[t \mathrm{~s}(22) \geq 2.11]$. It appears that a sweet taste is sufficient to demonstrate conditioned hypodipsia.

\section{GENERAL DISCUSSION}

The rats that received a novel taste CS (saccharin, saline, quinine, or sucrose) paired with an emetic US (LiCl) showed a substantial decrease in consumption of that taste CS, relative to their initial consumption and to controls that had the taste explicitly unpaired with $\mathrm{LiCl}$. Initially, consumption of the paired taste increased with each nonreinforced presentation (i.e., extinction). Even after nine extinction trials, the rats that had had the saccharin CS paired with $\mathrm{LiCl}$ consumed less of the saccharin solution than did controls (i.e., conditioned hypodipsia). A similar data pattern was observed with a sucrose taste CS (Experiment 4). Rosas and Bouton (1996) were the first to report the conditioned hypodipsia effect with saccharin as the taste CS. They concluded that extinction does not completely destroy the excitatory association between the saccharin CS and the lithium US. Our demonstration of this effect with saccharin, extended to sucrose, 
is consistent with this retained aversion interpretation. These results, in fact, challenge accounts of extinction based on the complete loss or unlearning of the excitatory CS-US association (Rescorla \& Wagner, 1972; Skinner, 1938).

On the other hand, we did not find conditioned hypodipsia with two different concentrations of saline ( $1 \%$ or $0.5 \%$ ) or with two different concentrations of quinine $(0.005 \%$ or $0.001 \%)$. After extinction, LiCl-paired rats drank these tastes at a level comparable with that of controls. These results suggest that a bitter taste and a salty taste quality were neither necessary nor sufficient for the expression of conditioned hypodipsia in the present experimental situation. The results of Experiments 1 and 4, in contrast, argue that a sweet (or preferred) taste quality may be sufficient to demonstrate conditioned hypodipsia. Why should a conditioned aversion be retained with a sweet taste but not with a bitter or salty taste?

One possibility to consider is that the rats in our experimental situation may have developed over trials (without $\mathrm{LiCl}$ ) a preference for the sweet tastes, but not for the salty or the bitter tastes. If this notion is true, rats that receive a sweet solution in the control condition should show a preference over water for that solution in a two-bottle test; controls that receive quinine or saline should not show a preference. In just such a pilot study, we found that sucrose-exposed controls preferred the $10 \%$ sucrose solution to water (proportion of sucrose intake $=.79 \pm .10$ ). Controls that received the $0.001 \%$ quinine taste avoided that solution in the two-bottle test (proportion of quinine intake $=.27 \pm .12$ ). Controls also avoided the $1 \%$ saline solution (proportion of saline intake $=.20 \pm .10$ ). In contrast, controls that received the $0.5 \%$ saline solution drank comparable amounts of water and saline (proportion of saline intake $=.56 \pm .06$ ).

The above discussion suggests that the development of a preference in the control group may be necessary for demonstrating conditioned hypodipsia. Thus, rather than attributing group differences to the expression of a retained aversion, this alternative account argues that the conditioning experience prevents the later development of a preference in paired rats. There are several interesting implications of this preference hypothesis. First, it suggests a partial dissociation between acquisition of a preference and acquisition of an aversion. That is, the expression of an aversion is more susceptible to extinction, but the process(es) that prevents the development of a preference is not. Rosas and Bouton (1996) found that saccharin consumption in lithium-paired rats went to a neutral point after extensive extinction. Although our measure of intake does not presume a neutral point (see the Data Analysis section of Experiment 1), consumption of saccharin and sucrose returned to initial consumption levels after extinction.

This preference hypothesis makes clear a priori predictions as to when the conditioned hypodipsia effect will be obtained - that is, any time the taste CS is demonstrably preferred to water under the conditions of the exper- iment. Future experimentation, however, will have to determine whether conditioned hypodipsia is specific to preferred sweet tastes, or whether other preferred tastes also maintain a conditioned hypodipsia. Indeed, in pilot experiments, we were hoping to find a saline concentration that would be preferred by the unpaired control. Previous research using vastly different fluid access procedures (one 15-min access period per $24 \mathrm{~h}$ ) found that a $0.3 \%$ concentration of saline was preferred over water in the control condition (Bevins, Delzer, \& Bardo, 1997). However, the rats that received the control procedures used in the present report consumed similar amounts of a $0.3 \%$ saline solution and water in a two-bottle test (proportion of saline intake $=.50 \pm .13$; cf. to $0.5 \%$ saline).

The preference hypothesis of conditioned hypodipsia is compatible with the view that the excitatory CS-US association remains, at least in part, intact after extinction. Indeed, a critical assumption of this hypothesis is that some aspect of the conditioned taste aversion experience must remain intact. It is this retained experience that prevents the development of a preference. From our perspective, it is of interest to determine the nature of this retained experience and to examine the conditions under which this experience is altered. For example, how permanent is the conditioned hypodipsia effect? Would further taste-alone trials result in the paired group returning to control levels? It may be that conditioned aversions to sweet/preferred tastes extinguish slower than those to bitter and salty tastes in our situation (i.e., extinction was incomplete). Also, what effect would taste preexposure have on the conditioned hypodipsia effect? Preexposure may weaken the aversive conditioning; however, preexposure may also allow a preference to develop. It is not clear whether a preference would redevelop to a preexposed taste that was conditioned, then extinguished.

\section{REFERENCES}

BARKER, L. M. (1976). CS duration, amount, and concentration effects in conditioning taste aversions. Learning \& Motivation, 7, 265-273.

Bennett, C. H., Tremain, M., \& Mackintosh, N. J. (1996). Facilitation and retardation of flavour aversion conditioning following prior exposure to the CS. Quarterly Journal of Experimental Psychology, 49B, $220-230$.

Bevins, R. A., Delzer, T. A., \& Bardo, M. T. (1997). Unexpressed morphine conditioned salt aversion: Procedural variants and hypertonicity of salt. Behavioural Processes, 40, 129-136.

Bond, N., \& Di Giusto, E. (1975). Amount of solution drunk is a factor in the establishment of taste aversion. Animal Learning $\& B e$ havior, 3, 81-84.

Bouton, M. E. (1991). Context and retrieval in extinction and in other examples of interference in simple associative learning. In L. W. Dachowski \& C. F. Flaherty (Eds.), Current topics in animal learning: Brain, emotion, and cognition (pp. 25-53). Hillsdale, NJ: Erlbaum.

DELAMATER, A. R. (1996). Effects of several extinction treatments upon the integrity of Pavlovian stimulus-outcome associations. Animal Learning \& Behavior, 24, 437-449.

DESs, N. K. (1993). Saccharin's aversive taste in rats: Evidence and implications. Neuroscience \& Biobehavioral Reviews, 17, 359-372.

Miller, R. R., \& Oberling, P. (1998). Analogies between occasion setting and Pavlovian conditioning. In N. A. Schmajuk \& P. C. Holland (Eds.), Occasion setting: Associative learning and cognition in animals (pp. 3-35). Washington, DC: American Psychological Association. 
Nolan, L. J., McCaughey, B. K., Giza, B. K., Rhinehart-Doty, J. A., Smith, J. C., \& ScotT, T. R. (1997). Extinction of a conditioned taste aversion in rats: 1. Behavioral effects. Physiology \& Behavior, 61, 319-323

Parker, L. A., \& Revusky, S. (1982). Generalized conditioned flavor aversions: Effects of toxicosis training with one flavor on the preference for different novel flavors. Animal Learning \& Behavior, 10, 505-510.

Pavlov, I. P. (1927). Conditioned reflexes: An investigation of the physiological activity of the cerebral cortex (G. V. Anrep, Trans.). London: Oxford University Press.

RESCORLA, R. A. (1993). Inhibitory associations between $S$ and $R$ in extinction. Animal Learning \& Behavior, 21, 327-336.

ResCorl,A, R. A. (1997). Spontaneous recovery after Pavlovian conditioning with multiple outcomes. Animal Learning \& Behavior, 25, 99-107.

RESCORLA, R. A., \& WAGNER, A. R. (1972). A theory of Pavlovian conditioning: Variations in the effectiveness of reinforcement and nonreinforcement. In A. H. Black \& W. F. Prokasy (Eds.), Classical conditioning: 11. Current research and theory (pp. 64-99). New York: Appleton-Century-Crofts.
RoBbins, S. J. (1990). Mechanisms underlying spontaneous recovery in autoshaping. Journal of Experimental Psychology: Animal Behavior Processes, 16, 235-249.

Rosas, J. M., \& Bouton, M. E. (1996). Spontaneous recovery after extinction of a conditioned taste aversion. Animal Learning \& Behavior, 24, 341-348.

SKINNER, B. F. (1938). The behavior of organisms. New York: AppletonCentury-Crofts.

\section{NOTE}

1. Exclusion of this water recovery day did not affect the major results of interest. In subsequent research not reported here, the water recovery day was included in the experiment, and no difference between the paired and unpaired groups was found in quinine consumption after extinction.

(Manuscript received February 4, 1999; revision accepted for publication March 4, 1999.)

\section{0th Annual Meeting of the Psychonomic Society Los Angeles, California November 18-21, 1999}

The 40th Annual Meeting of the Psychonomic Society will be held in Los Angeles, California, at the Century Plaza Hotel. Registration will begin on Thursday evening, November 18. A poster session will be held on Thursday evening; spoken sessions will begin on the morning of Friday, November 19. Sessions will continue through noon on Sunday, November 21.

Programs and hotel reservation cards were mailed to members and associates in the beginning of September. Additional programs will be available at the meeting registration desk for $\$ 10.00$.

For further information, please contact the Secretary-Treasurer of the Society: Roger L. Mellgren, Department of Psychology, Box 19528, University of Texas, Arlington, TX 76019-0528 (phone: 817-272-2775; fax: 817-272-2364; e-mail: mellgren@uta.edu). 